\title{
Desempenho agronômico de cultivares de alface em aquaponia
}

\author{
Agronomic performance of lettuce cultivars in aquaponics \\ Comportamiento agronómico de cultivares de lechuga en acuaponía
}

Recebido: 12/07/2021 | Revisado: 21/07/2021 | Aceito: 24/07/2021 | Publicado: 01/08/2021

Fábio Teixeira de Carvalho Mendes

ORCID: https://orcid.org/0000-0001-5629-0340

Universidade Vale do Rio Verde, Brasil

E-mail: fabioteixeiratc@outlook.com

Aurivan Soares de Freitas

ORCID: https://orcid.org/0000-0002-5322-3676

Universidade Vale do Rio Verde, Brasil

E-mail: aurivan.soares@hotmail.com

Eliana Alcantra

ORCID: https://orcid.org/0000-0001-9292-3256 Universidade Vale do Rio Verde, Brasil

E-mail: lialcantra@yahoo.com.br

Rosângela Francisca de Paula Vitor Marques

ORCID: https://orcid.org/0000-0001-6646-0809 Universidade Vale do Rio Verde, Brasil

E-mail: rosangela.marques@unicor.edu.br

Alisson Souza de Oliveira

ORCID: https://orcid.org/0000-0001-7885-9542 Universidade Vale do Rio Verde, Brasil

E-mail: alissonso@hotmail.com

Ronei Aparecido Barbosa

ORCID: https://orcid.org/0000-0002-4850-7244

Instituto Federal de Educação, Ciência e Tecnologia do Sul de Minas, Brasil E-mail: roneibarbosa50@gmail.com

Mairon Cézar de Pádua

ORCID: https://orcid.org/0000-0001-9716-0470 Universidade Vale do Rio Verde, Brasil E-mail: maironcpadua@hotmail.com

Raphael Ribeiro Junqueira

ORCID: https://orcid.org/0000-0002-6856-5548 Universidade Vale do Rio Verde, Brasil

E-mail: rafaelzao94@hotmail.com

\section{Resumo}

A alface é uma hortaliça que pode ser produzida em diferentes sistemas de cultivo, como é caso da aquaponia. $\mathrm{O}$ objetivo do trabalho foi avaliar o desempenho de cultivares de alface dos grupos roxa, americana, lisa e crespa de alface em aquaponia. $\mathrm{O}$ sistema foi composto por uma área de aproximadamente $10 \mathrm{~m}^{3}$, sendo composto de um tanque de criação de peixes de $1 \mathrm{~m}^{3}$, um filtro decantador de $240 \mathrm{~L}$, um filtro biológico de $240 \mathrm{~L}$ e nove canaletas de PVC de $3 \mathrm{~m}$ de comprimento. $\mathrm{O}$ experimento foi composto por quatro tratamentos, os quais consistiram por quatro grupos de cultivares de alface: roxa (Carmim); americana (Manteiga); lisa (Elisa) e; crespa (Grand Rapids). As variáveis analisadas foram massa fresca, comprimento da raiz e do caule e número de folhas. Aos 20 dias, a maior massa fresca foi constatada na cultivar crespa $(121,22 \mathrm{~g})$. Para o comprimento da raiz as cultivares crespas e americanas demonstraram maiores médias com 16,22 cm. O comprimento do caule foi maior nas cultivares lisa (11,88 cm) e crespa $(12,77 \mathrm{~cm})$. Com 19,33 folhas, o maior número foi encontrado na cultivar lisa. Já aos 40 dias, a cultivar americana apresentou maior massa fresca $(321,33 \mathrm{~g})$ e comprimento da raiz $(20,55 \mathrm{~cm})$. Já para o comprimento do caule, a cultivar crespa apresentou maior valor $(17,88 \mathrm{~cm})$. Para o número de folhas, as cultivares americana $(34,22)$, roxa $(33)$ e lisa $(32,22)$ apresentaram maiores valores. Portanto, em aquaponia há diferença significativa entre as cultivares de alface dos grupos roxa, americana, lisa e crespa.

Palavras-chave: Lactuca sativa L; Tilápia; Cultivo sem solo; Oreochromis niloticus.

\section{Abstract}

Lettuce is a vegetable that can be produced in different cultivation systems, such as aquaponics. The objective of this work was to evaluate the performance of lettuce cultivars from the purple, american, smooth and curly lettuce groups in aquaponics. The system consisted of an area of approximately $10 \mathrm{~m}^{3}$, consisting of a $1 \mathrm{~m}^{3}$ fish breeding tank, a 240 $\mathrm{L}$ settling filter, a $240 \mathrm{~L}$ biological filter and nine $3 \mathrm{~m}$ long PVC channels. The experiment consisted of four 
treatments, which consisted of four groups of lettuce cultivars: purple (Carmine); American (Butter); lisa (Elisa) and; curly (Grand Rapids). The variables analyzed were fresh mass, length of root and stem and number of leaves. At 20 days, the highest fresh mass was found in the crisp cultivar (121.22g). For root length, the curly and American cultivars showed higher averages with $16.22 \mathrm{~cm}$. Stem length was greater in smooth $(11.88 \mathrm{~cm})$ and curly $(12.77 \mathrm{~cm})$ cultivars. With 19.33 leaves, the largest number was found in the smooth cultivar. At 40 days, the cultivar Americana presented higher fresh mass $(321.33 \mathrm{~g})$ and root length $(20.55 \mathrm{~cm})$. As for the length of the stem, the curly cultivar presented the highest value $(17.88 \mathrm{~cm})$. For the number of leaves, cultivars americana $(34.22)$, purple (33) and smooth (32.22) showed higher values. Therefore, in aquaponics there is a significant difference between lettuce cultivars from the purple, american, smooth and curly groups.

Keywords: Lactuca sativa L; Tilapia; Soil-free cultivation; Oreochromis niloticus.

\section{Resumen}

La lechuga es una hortaliza que se puede producir en diferentes sistemas de cultivo, como la acuaponía. El objetivo de este trabajo fue evaluar el comportamiento de cultivares de lechuga de los grupos de lechugas moradas, americanas, lisas y crujientes en acuaponía. El sistema constaba de un área de aproximadamente $10 \mathrm{~m} 3$, compuesto por un tanque de cría de peces de $1 \mathrm{~m} 3$, un filtro decantador de $240 \mathrm{~L}$, un filtro biológico de $240 \mathrm{~L}$ y nueve canales de PVC de $3 \mathrm{~m}$ de longitud. El experimento consistió en cuatro tratamientos, que consistieron en cuatro grupos de cultivares de lechuga: púrpura (Carmín); Americano (mantequilla); lisa (Elisa) y; rizado (Grand Rapids). Las variables analizadas fueron masa fresca, longitud de raíz y tallo y número de hojas. A los 20 días, la masa fresca más alta se encontró en el cultivar crujiente $(121,22 \mathrm{~g})$. Para la longitud de la raíz, los cultivares rizado y americano mostraron promedios más altos con $16.22 \mathrm{~cm}$. La longitud del tallo fue mayor en los cultivares lisos $(11,88 \mathrm{~cm})$ y rizados $(12,77 \mathrm{~cm})$. Con 19,33 hojas, el mayor número se encontró en el cultivar liso. A los 40 días, el cultivar Americana presentó mayor masa fresca $(321.33 \mathrm{~g})$ y longitud de raíz $(20.55 \mathrm{~cm})$. En cuanto a la longitud del tallo, el cultivar rizado presentó el valor más alto (17,88 cm). Para el número de hojas, los cultivares americana (34.22), morado (33) y liso (32.22) mostraron valores más altos. Por lo tanto, en acuaponía existe una diferencia significativa entre los cultivares de lechuga de los grupos morado, americano, liso y rizado.

Palabras clave: Lactuca sativa L; Tilapia; Cultivo sin suelo; Oreochromis niloticus.

\section{Introdução}

A alface (Lactuca sativa L.) é uma hortaliça anual, originária de clima temperado, pertencente à família Asteracea, sendo uma das hortaliças mais populares e consumidas mundialmente (Nick \& Borém, 2019). Segundo Henz et al. (2009) quase todas as cultivares de alface desenvolvem-se bem em climas amenos, principalmente no período de crescimento vegetativo. Para estes autores a ocorrência de temperaturas mais elevadas acelera o ciclo cultural e, dependendo do genótipo, pode resultar em plantas menores em razão do pendoamento ser mais precoce.

As cultivares de alface mais conhecidas e consumidas no Brasil pertencem aos grupos crespas e as lisas, sendo que algumas dessas cultivares foram melhoradas com o intuito de serem cultivadas no verão ou adaptadas para regiões tropicais, com temperatura e pluviosidade elevadas (Henz et al., 2009). Recentemente apareceram as cultivares pertencentes aos grupos roxa e com as folhas frisadas (Henz et al., 2009). Todavia, as cultivares destes grupos podem variar quanto à duração do período vegetativo, florescimento, número de folhas e massa da planta (Lêdo, 1998).

A alface pode ser produzida em diferentes sistemas de cultivo, tais como orgânico, convencional e hidropônico. Recentemente, uma técnica nova técnica tem surgido com maiores vantagens, trata-se da aquaponia. Esta técnica tem apresentado resultados mais vantajosos, pois além de proporcionar a produção de espécies vegetais, permite a criação de peixes (Carvalho et al., 2017; Rocha et al., 2017).

A integração da aquicultura e da hidroponia foi criada para a produção de alimentos usando o sistema de recirculação da água e de nutrientes. A aquaponia tem como vantagens a produção de alimentos saudáveis e com menor impacto ao meio ambiente (Diver, 2006; Hundley et al., 2013; Mateus, 2009). Assim, aquaponia surge como uma alternativa de intensificação da produção de maneira sustentável (Belintano et al., 2020). Esta técnica é uma das soluções para o aumento da produção frente ao crescimento da população mundial (Goddek et al., 2019; Sátiro et al., 2018).

A aquaponia consiste em um sistema de produção de peixes e vegetais, utilizando a mesma água em um processo de recirculação. Basicamente, os dejetos produzidos pelos peixes atuam na fonte de nutrientes dos vegetais, que por sua vez, 
devolvem a água aos peixes com uma maior qualidade. Produzindo assim, alimentos com economia de água e controle total do efluente produzido (Hundley et al., 2013).

Atualmente, sabe-se que é possível produzir diversas espécies vegetais em aquaponia como alface, manjericão, agrião, repolho, rúcula, morango, pimenta, tomate, quiabo e pepino. As espécies vegetais adaptadas à hidroponia são sempre recomendadas para a aquaponia. Sendo assim, deve-se avaliar apenas necessidades e limitações das plantas relacionadas a espaço, nutrição, aeração, temperatura e radiação solar (Carneiro et al., 2015; Martins, 2017).

Na literatura, informações sobre o desenvolvimento de cultivares em aquaponia são escassas. Sendo assim, objetivouse com este trabalho avaliar o desempenho de cultivares dos grupos roxa, americana, lisa e crespa de alface cultivadas em sistema aquapônico.

\section{Metodologia}

O experimento foi realizado no Sítio São João na cidade de Três Corações, Minas Gerais. Clima tropical, de inverno seco e verão chuvoso. Com pluviosidade anual em média de $1.400 \mathrm{~mm}$ por ano. Temperatura média de $20,2{ }^{\circ} \mathrm{C}$ e altitude de 839 metros.

Para o desenvolvimento do trabalho foi instalado um sistema de aquaponia seguindo as recomendações de Carneiro et al. (2016). O sistema apresentado ocupou uma área de aproximadamente $10 \mathrm{~m} 2$, sendo composto de um tanque de criação de peixes de $1 \mathrm{~m} 3$, um filtro decantador de $240 \mathrm{~L}$, um filtro biológico de $240 \mathrm{~L}$, nove canaletas de PVC de $3 \mathrm{~m}$ de comprimento para o cultivo das plantas. Foi utilizado 250 alevinos de tilápia (Oreochromis niloticus) em uma caixa com capacidade para 1000 litros.

A alimentação dos peixes foi realizada 2 vezes ao dia. A limpeza do sistema foi realizada no mínimo uma vez por semana, para evitar o entupimento das tubulações. As mudas foram produzidas em bandejas de isopor de 200 células. As mudas foram transferidas para o sistema 30 dias após a semeadura.

O experimento foi composto por quatro tratamentos, os quais consistiram por quatro grupos de cultivares de alface: roxa (Carmim); americana (Manteiga); lisa (Elisa) e; crespa (Grand Rapids). O ensaio foi conduzido em delineamento inteiramente casualizado (DIC), com 3 repetições. Cada repetição consistiu por uma planta.

Foram realizadas duas avaliações aos 20 e aos 40 dias. As variáveis analisadas foram comprimento da raiz e do caule $(\mathrm{cm})$, número de folhas e massa fresca das plantas $(\mathrm{g})$. O comprimento da raiz foi medido por uma régua graduada. Em seguida as plantas foram pesadas em balança digital para obtenção da massa fresca. Logo após, contou-se o número de folhas. Por fim, na avaliação do comprimento do caule cortou-se a cabeça da alface longitudinalmente, assim, possibilitando a exposição do caule, em que se efetuou a medição, com uso de uma régua (Mota et al., 2003).

Os dados foram submetidos à análise de variância, e quando significativos foram submetidos ao teste de Scott-Knott a 5\% de probabilidade. As análises foram realizadas utilizando o programa Sisvar (Ferreira, 2011).

\section{Resultados e Discussão}

Houve diferença significativa entre as cultivares de alface nas duas épocas analisadas.

\subsection{Análises realizadas aos 20 dias}

Nas análises realizadas aos 20 dias foi observado que para massa fresca das plantas a cultivar que apresentou maior valor foi a crespa com 121,22g, seguida das cultivares americana e lisa, sendo que o menor resultado se constatou na cultivar roxa com 52,55 g (tabela 1). Em estudo realizado em sistema convencional com diversas cultivares crespas, observou-se que as cultivares que apresentaram os maiores valores médios de massa fresca de plantas foram Vanda e Verônica com 776,68g e 
682,5g, respectivamente (Suinaga et al., 2013). Estes resultados justifica a importância de se avaliar o desempenho de cultivares em condições específicas, como é caso do presente estudo.

Para o comprimento da raiz, as cultivares crespas e americanas demonstraram maiores médias com $16,22 \mathrm{~cm}$. Já as cultivares Roxa e lisa apresentaram 9,22g e 8,77g, respectivamente (tabela 1). O comprimento das raízes nas cultivares dos grupos crespas e americanas, se assemelham com os $16,5 \mathrm{~cm}$ de comprimento verificados por Monteiro Filho et al. (2014) ao estudarem cultivares de alface (Crespa, Manteiga e Rubi) em soluções nutritivas com 100\% de solução mineral.

Em relação ao comprimento do caule, as cultivares lisa e crespa com 11,88 cm e 12,77 cm, respectivamente, tiveram os maiores resultados. Avaliando a divergência genética de alface cultivada sob sistema hidropônico, Oliveira et al. (2004) verificaram que as cultivares Brisa, Itapuã-401, Marisa, Salad Bowl (grupo mimosa), destacaram-se quanto ao peso de matéria fresca da planta, mas apresentaram menor tolerância ao calor, verificada pelo maior comprimento do caule $(14,2 \mathrm{~cm})$.

Por fim, o número de folhas foi maior na cultivar lisa com valor de 19,33 folhas, por outro lado a cultivar americana com 9,22 folhas teve o menor número (Tabela 1). Resultados semelhantes foram encontrados por Silva et al. (2007), ao evidenciarem que a cultivar Regina (do grupo lisa), com 47 folhas, foi superior aos grupos crespa (Mônica), grupo mimosa rocha (Salad Browm) e americana (Tainá). O número de folhas maior que os encontrado neste trabalho deve-se ao fato de que as análises foram realizadas no final do experimento. No Quadro 2, observa-se que o número de folhas aos 40 dias se aproximou dos encontrados por estes autores.

Tabela 1. Massa fresca $(\mathrm{g})$, comprimento da raiz $(\mathrm{cm})$, comprimento do caule $(\mathrm{cm})$ e número de folhas de diferentes grupos/cultivares de alface crescida em aquaponia aos 20 dias.

\begin{tabular}{lcccc}
\hline & Massa fresca & Comprimento da raiz & Comprimento do caule & Número de folhas \\
\cline { 2 - 4 } Grupos/Cultivares & $----g----$ & ----------- & \\
Roxa/Carmim & $52,55 \mathrm{c}$ & $9,22 \mathrm{~b}$ & $7,55 \mathrm{~b}$ & $14,11 \mathrm{~b}$ \\
Americana/Manteiga & $87,44 \mathrm{~b}$ & $16,22 \mathrm{a}$ & $7,55 \mathrm{~b}$ & $9,22 \mathrm{c}$ \\
Lisa/Elisa & $80,55 \mathrm{~b}$ & $8,77 \mathrm{~b}$ & $11,88 \mathrm{a}$ & $19,33 \mathrm{a}$ \\
Crespa/Grand Rapids & $121,22 \mathrm{a}$ & $16,22 \mathrm{a}$ & $12,77 \mathrm{a}$ & $13,33 \mathrm{~b}$
\end{tabular}

Médias seguidas pela mesma letra na coluna, não diferem estatisticamente por meio do teste de Scott-Knott, a 5\% de probabilidade. Fonte: Autores.

\subsection{Análises realizadas aos 40 dias}

Já para as análises realizadas aos 40 dias, a cultivar americana apresentou maior massa fresca $(321,33 \mathrm{~g})$, seguida pela cultivar crespa com 282,44g. Para esta variável, as cultivares roxa $(204,77 \mathrm{~g})$ e lisa $(204,22 \mathrm{~g})$ manifestaram menor valor (tabela 2). A cultivar de alface Verônica (grupo crespa) em hidroponia com água salina apresentou crescimento superior a cultivar Pira Roxa (grupo roxa) com 37,71\%, 20,58\% e 35,80\% para massa fresca das folhas, massa fresca do caule e massa fresca da parte aérea, respectivamente (Paulus et al., 2010). A maior massa da alface americana pode estar relacionada com as características da própria cultivar e não em relação a influência da aquaponia. Em estudo realizado com cultivares deste grupo nos sistemas túnel baixo de polietileno e campo aberto, constatou-se que os cultivares Rubette e Grandes Lagos 659 apresentaram as maiores alturas de plantas com 20,27 cm e 18,70 cm, respectivamente (Brzezinski et al., 2017). Segundo os autores, possivelmente, isso ocorreu em razão das características genéticas distintas de cada cultivar, em relação ao tamanho médio das plantas.

Em relação ao comprimento da raiz, a alface americana novamente apresentou maior valor $(20,55 \mathrm{~cm})$, seguida pelas cultivares crespa $(15,00 \mathrm{~g})$ e lisa $(14,44 \mathrm{~g})$, sendo que a roxa apresentou menor comprimento com $10,33 \mathrm{~cm}$. Já para o 
comprimento do caule, a cultivar crespa manifestou maior valor com $17,88 \mathrm{~cm}$ seguida pela roxa $(15,33 \mathrm{~cm})$. A cultivar lisa teve um resultado intermediário $(12,44 \mathrm{~cm}$ ). E por fim, o menor valor foi observado na cultivar americana, com 6,0 cm (Tabela 2). Estes resultados são semelhantes aos constatados por Henrique (2020) ao evidenciarem que as cultivares Milena (crespa) e Lucy Brown (americana) proporcionaram maior comprimento de raiz com $41,2 \mathrm{~cm}$.

No tocante ao número de folhas, as cultivares americana, roxa e lisa foram superiores e estatisticamente iguais apresentando valores de 34,22, 33,00 e 32,22, respectivamente. Por outro lado, a cultivar crespa proporcionou menor número com 23,66 folhas (tabela 2). Em estudo realizado objetivando avaliar cultivares dos grupos dos grupos americana, lisa e crespa, em sistema hidropônico-NFT, foi constatado que para as cultivares do grupo americana não houve diferença estatística. Entretanto, neste mesmo estudo no grupo lisa, as cultivares Regina 440 com 39,3 folhas e Brasil 303, com 36,3 folhas apresentaram maiores valores (Sediyama et al., 2009). No grupo crespa, as cultivares vera e verônica com 21,8 e 21,2 tiveram os menores desempenhos, respectivamente (Sediyama et al., 2009). Avaliando cultivares americanas em solo e em hidroponia, no inverno e verão, Feltrim et al. (2005) evidenciaram que o número de folhas foi influenciado pelo fator cultivar. A cultivar Lorca apresentou o maior número de folhas sem, no entanto, diferir significativamente da cultivar Lucy Brown, enquanto a cultivar Mesa 659 resultou em 75\% do número de folhas da cultivar Lorca.

No presente trabalho, os menores valores constatados para a maioria das variáveis na cultivar Carmim (grupo roxa) (tabelas 1 e 2), não significa que esta não possa ser cultivada em aquaponia, pois possivelmente é uma característica da própria cultivar. Em um sistema análogo a aquaponia, denominado tecnologia de produção em sistema de bioflocos (BFT) foi constatado que os comprimentos das folhas das cultivares roxa $(42,12 \mathrm{~cm})$ e crespa $(43,71 \mathrm{~cm})$ foram estatisticamente superiores a cultivar lisa que apresentou valor de $28,62 \mathrm{~cm}$ (Lenz et al., 2018). Além disso, neste trabalho, os autores observaram que no sistema de água salobra a cultivar roxa foi a única que recebeu notas "A" em termos de qualidade de plantas.

Tabela 2. Massa fresca $(\mathrm{g})$, comprimento da raiz $(\mathrm{cm})$, comprimento do caule $(\mathrm{cm})$ e número de folhas de diferentes grupos/cultivares de alface crescida em aquaponia aos 40 dias.

\begin{tabular}{lcccc}
\hline & Massa fresca & Comprimento da raiz & Comprimento do caule & Número de folhas \\
\cline { 2 - 4 } Grupos/Cultivares & $----g----$ & ---------- & \\
Roxa/Carmim & $204,77 \mathrm{c}$ & $10,33 \mathrm{c}$ & $15,33 \mathrm{~b}$ & $33,00 \mathrm{a}$ \\
Americana/Manteiga & $321,33 \mathrm{a}$ & $20,55 \mathrm{a}$ & $6,00 \mathrm{~d}$ & $34,22 \mathrm{a}$ \\
Lisa/Elisa & $204,22 \mathrm{c}$ & $14,44 \mathrm{~b}$ & $12,44 \mathrm{c}$ & $32,22 \mathrm{a}$ \\
Crespa/Grand Rapids & $282,44 \mathrm{~b}$ & $15,00 \mathrm{~b}$ & $17,88 \mathrm{a}$ & $23,66 \mathrm{~b}$ \\
\hline
\end{tabular}

Médias seguidas pela mesma letra na coluna, não diferem estatisticamente por meio do teste de Scott-Knott, a 5\% de probabilidade. Fonte: Autores.

Em outro estudo, comparando o sistema hidropônico, com os sistemas de aquaponia com a espécie de peixe Rhamdia quelen e o de aquaponia com bioflocos, verificou-se que para a maioria das variáveis o sistema hidropônico apresentou menor valor. Por exemplo, para o peso fresco, os valores foram 2,6g, 24,6 e 39,8 g, respectivamente. Nesta mesma ordem, os valores para comprimento de raiz foram 7,4 cm, $42 \mathrm{~cm}$ e 39,3 cm (Rocha et al., 2017).

Apesar das escassas informações sobre aquaponia na literatura, entende-se que a técnica pode ser adotada dentro de um sistema sustentável de produção e que pode permitir que as plantas utilizem os nutrientes provenientes da água do cultivo de peixes, melhorando a qualidade da água (Hundley et al., 2013). No presente estudo, as cultivares dos grupos crespa e americana se sobressaíram em relação as cultivares dos grupos roxa e a lisa, entretanto, mesmo assim houve desenvolvimento 
destas últimas. Cabe ressaltar que o sistema aquapônico se assemelha com o sistema hidropônico, já que em ambos, as plantas são cultivas em sistemas de recirculação de água (Hundley et al., 2013).

No presente trabalho, por meio das variáveis massa fresca, comprimento da raiz e do caule e produção de folhas, ficou evidente que na aquaponia é possível produzir as cultivares dos grupos roxa, americana, lisa e crespa. Em hidroponia, com a técnica do NFT (Nutrient Film Technique ou fluxo laminar de solução), constatou-se que as cultivares dos grupos lisa (Aurora, Lívia e Regina) e crespa (Brisa, Mimosa e Verônica) mantiveram a qualidade nutricional semelhante a alface produzido no sistema convencional (Ohse et al., 2001). Para os autores, não houve diferença entre as cultivares hidropônicas e convencionais para teores de água, extrato etéreo, carboidratos totais, proteína e valor calórico. Dessa forma, como a aquaponia possui características semelhantes a hidroponia, pode-se propor que a técnica é promissora no cultivo da alface, garantindo a quantidade e a qualidade da produção, sendo então mais opção para os produtores.

\section{Conclusão}

Aos 20 dias de cultivo em aquaponia, os maiores valores para massa fresca, comprimento da raiz, comprimento do caule são encontrados nas cultivares dos grupos crespa $(121,22 \mathrm{~cm})$, americana e crespa $(16,22 \mathrm{~cm})$, lisa $(11,88 \mathrm{~cm})$ e crespa $(12,77 \mathrm{~cm})$ e lisa $(19,33)$, respectivamente.

Aos 40 dias de cultivo em aquaponia os maiores valores para massa fresca, comprimento da raiz, comprimento do caule são observados nas cultivares dos grupos americana $(321,33 \mathrm{~cm})$, americana $(20,55 \mathrm{~cm})$, crespa $(18,88 \mathrm{~cm})$ e americana $(34,22)$, roxa $(33)$ e lisa $(32,22)$, respectivamente.

\section{Referências}

Belintano, A. L. O., Kreutz, F. I., Messias, E. A., Ibanhez, J. R., Ferreira, M. W. \& Guilherme, D. O. (2019). Sistema de aquaponia em escada: um estudo de caso. Pubvet, 14(1), 1-9. 10.31533/pubvet.v14n1a500.1-9

Brzezinski, C. R., Abati, J., Geller, A., Werner, F. \& Zucareli, C. (2017). Produção de cultivares de alface americana sob dois sistemas de cultivo. Revista Ceres, 64, 83-89. 10.1590/0034-737X201764010012

Carneiro, P. C. F., Morais, C. A. R. S., Nunes, M. U. C., Maria, A. N. \& Fujimoto, R. Y. (2015). Produção integrada de peixes e vegetais em aquaponia. Embrapa Tabuleiros Costeiros-Documentos-Aracaju, Aracaju: Embrapa Tabuleiros Costeiros, ISSN 1678-1937, 189.

Carneiro, P., Maria, A., Fujimoto, R. \& Nunes, M. (2016). Sistema familiar de aquaponia em canaletas. Embrapa Tabuleiros Costeiros-Circular Técnica (INFOTECA-E).

Carvalho, A. R., Brum, O. B., Chimóia, E. P., \& Figueiró, E. A. G. (2017). Avaliação da produtividade da aquaponia comparada com a hidroponia convencional. Rev. Vivência, 13(24), 79-91.

Diver, S. Aquaponics - Integration of hydroponic swith aquaculture. National Sustainable Agriculture Information Service, 2006. 28p.

Feltrim, A. L., Cecílio Filho, A. B., Branco, R. B., Barbosa, J. C. \& Salatiel, L. T. (2005). Produção de alface americana em solo e em hidroponia, no inverno e verão, em Jaboticabal, SP. Revista Brasileira de Engenharia Agrícola e Ambiental, 9(4), 505-509. 10.1590/S1415-43662005000400010

Ferreira, D. F. Sisvar: a computer statistical analysis system. (2011). Ciência e Agrotecnologia, 35(6), 1039-1042, 10.1590/S1413-70542011000600001

Goddek, S., Joyce, A., Kotzen, Benz. \& Burnell, G. M. (2019). Aquaponics Food Production Systems. (eBook). 10.1007/978-3-030-15943-6

Henrique, A. G. S. (2020). Avaliação do desempenho de cultivares de alface em sistema hidropônico. https://repositorio.ufscar.br/handle/ufscar/13007.

Henz, G. P. Suinaga, F. (2009). Tipos de Alface Cultivados no Brasil.

Hundley, G. C. \& Navarro, R. D (2013). Aquaponia: a integração entre piscicultura e a hidroponia. Revista Brasileira de Agropecuária Sustentável, 3(2), 52$61,10.21206 /$ rbas.v3i2.218

Lêdo, F. J. S. (1998). Diversidade genética e análise dialélica da eficiência nutricional para nitrogênio em alface (Lactuca sativa L.). 87p. (Tese doutorado).

Lenz, G. L., Durigon, E. G., Lapa, K. R. \& Emerenciano, M. G. C. (2018). Produção de alface (Lactuca sativa) em efluentes de um cultivo de tilápias mantidas em sistema BFT em baixa salinidade. Boletim do Instituto de Pesca, 43(4), 614-630. 10.20950/1678-2305.2017v43n4p614

Martins, P. (2017). AQUAPONIA, uma novidade na educação ambiental. Ambiental Mente Sustentable: Revista científica galego-lusófona de educación ambiental, 23, 101-106. 10.17979/ams.2017.23-24.0.3369 
Research, Society and Development, v. 10, n. 9, e50610918176, 2021

(CC BY 4.0) | ISSN 2525-3409 | DOI: http://dx.doi.org/10.33448/rsd-v10i9.18176

Mateus, J. (2009). Acuaponía: hidroponía y acuacultura, sistema integrado de producción de alimentos. Rev. Hidroponía Boletín, 44, 7-10.

Monteiro Filho, A. F., Pereira, G. L., Azevedo, M. R., Fernandes, J. D. \& de Azevedo, C. A. (2014). Cultivo hidropônico de cultivares de alface em soluções nutritivas organominerais otimizadas com a ferramenta SOLVER. Revista Brasileira de Engenharia Agrícola e Ambiental, 18 (4), 417-424. 10.1590/S141543662014000400009

Mota, J. H., Yuri, J. E., Freitas, S. A. C., Rodrigues Junior, J. C., Resende, G. M. \& Souza, R. J. (2003). Avaliação de cultivares de alface americana durante o verão em Santana da Vargem, MG. Horticultura Brasileira, 21(2), 234-237, 10.1590/S0102-05362003000200023

Nick, C. \& Borém, A. (2019). Alface do Plantio à Colheita.

Ohse, S., Dourado-Neto, D., Manfron, P.A. \& Santos, O. S. D. (2001). Qualidade de cultivares de alface produzidos em hidroponia. Scientia Agricola, 58(1), $181-185,10.1590 /$ S0103-90162001000100027

Oliveira, A. C. B., Sediyama, M. A. N., Pedrosa, M. W., Garcia, N. C. P. \& Garcia, S. L. R. (2004). Divergência genética e descarte de variáveis em alface cultivada sob sistema hidropônico. Acta Scientiarum. Agronomy, 26(2), 211-217. doi.org/10.4025/actasciagron.v26i2.1894

Paulus, D., Dourado Neto, D., Frizzone, J. A. \& Soares, T. M. (2010). Produção e indicadores fisiológicos de alface sob hidroponia com água salina. Horticultura Brasileira, 28(1), 29-35. 10.1590/S0102-05362010000100006

Rocha, A., Biazzetti Filho, M., Stech, M. \& Silva, R. P. (2017). Lettuce production in aquaponic and biofioc systems with silver catfish Rhamdia quelen. Bol Inst Pesca, 43, 64-73, 10.20950/1678-2305.2017.64.73

Sátiro, T. M., Neto, K. X. C. R. \& Delprete, S. E. (2018). Aquaponia: Sistema que integra produção de peixes com produção de vegetais de forma sustentável. Revista Brasileira de Engenharia de Pesca, 11(1), 38-54, 10.18817/repesca.v11i1.1513

Sediyama, M. A. N., Pedrosa, M. W., Salgado, L. T. \& Pereira, P. C. (2009). Desempenho de cultivares de alface para cultivo hidropônico no verão e no inverno. Científica, 37(2), 98-106. 10.15361/1984-5529.2009v37n2p98+-+106

Silva, M. L., Villela Junior, L. V. E., Colovatto, G. F. \& Sartori, R. A. (2007). Produção hidropônica de quatro cultivares de alface em Garça (SP). Revista Científica Eletrônica de Agronomia, 6(11), 1-7, 10.32406/v2n32019/76-81/agrariacad

Suinaga, F. A., Boiteux, L. S., Cabral, C. S. \& Rodrigues, C. D. S. (2013). Desempenho produtivo de cultivares de alface crespa. Embrapa Hortaliças-Boletim de Pesquisa e Desenvolvimento (INFOTECA-E). 